\title{
Reversibility and quantum coherence in one-dimensional quantum cellular automata
}

\author{
Federico Centrone, ${ }^{1}$ Camillo Tassi, ${ }^{2}$ Marco Barbieri, ${ }^{3}$ and Alessio Serafini ${ }^{4}$ \\ ${ }^{1}$ Dipartimento di Fisica, Sapienza Università di Roma, Piazzale Aldo Moro 5, 00185 Rome, Italy \\ ${ }^{2}$ Dipartimento di Matematica e Fisica, Università degli Studi Roma Tre, Via della Vasca Navale 84, 00146, Rome, Italy \\ ${ }^{3}$ Dipartimento di Scienze, Università degli Studi Roma Tre, Via della Vasca Navale 84, 00146 Rome, Italy \\ ${ }^{4}$ Department of Physics and Astronomy, University College London, Gower Street, London WC1E 6BT, England, United Kingdom
}

(Received 9 April 2018; published 3 July 2018)

\begin{abstract}
Quantum cellular automata are important tools in understanding quantum dynamics, thanks to their simple and effective list of rules. Here we consider a class of noisy, one-dimensional quantum cellular automata that allow one to shift from unitary dynamics to completely positive maps, investigating the evolution of coherence as well as the notion of reversibility in such a setting. To this aim, we associate an approximate reverse automaton to each noisy automaton, and assess its effect, and we define an irreversibility time based on the distance from the maximally mixed state, which is shown to be the only attractor of the automaton map in the presence of dephasing. Our analysis illustrates the interplay between unitary and noisy dynamics.
\end{abstract}

DOI: 10.1103/PhysRevA.98.012105

\section{INTRODUCTION}

Time is what is measured by a clock, which is anything performing a periodic motion. The periodicity is fundamental to quantify the passage of time, but time itself is revealed already as soon as anything moves at all. However, a clock does not give any preferred direction to the dynamic so, in principle, the notions of past and future might be regarded as those of left and right. Surprisingly, they are when dealing with isolated microscopic systems.

On the other hand, in our daily observation of nature, this symmetry is quite exceptional. Imagine, in fact, being able to observe the universe as time flows backwards. You would immediately notice that something is wrong and unnatural. Most of natural processes occur spontaneously, giving a precise direction to the stream of time [1-3]. The first attempt to face the problem of the emergence of an arrow of time in a macroscopic system was made by Boltzmann in his unification of mechanics and thermodynamics [4]. In his picture, a macroscopic system spends most of its time in its most probable configuration, which has the highest entropy value. Through the still debated generic mechanisms of equilibration and thermalization [5], physical spontaneous evolutions drive the system towards this configuration. If the initial state is already at the equilibrium, then on average the system has no dynamics, and time "stops."

For a many-body system, if the constituent parts start the dynamics in an initial uncorrelated state, they will begin to develop correlations through their interaction, producing an average increase in entropy and setting a precise direction to the arrow of time. This has been confirmed in various experiments in both classical [6] and quantum mechanics $[7,8]$, establishing a way to manipulate and control the flow of time operating on the system's initial conditions. However, understanding in general how these complex systems evolve can be a demanding task, due to the large number of parameters involved. Introducing test models is an effective way to gain insight on complexity by introducing minimal complications, while still maintaining a nontrivial phenomenology.

Cellular automata (CA) have been introduced in this context in the early days of computer science in order to investigate self-replicating systems, as well as other peculiar discrete dynamical occurrences [9]. Classical cellular automata are built as arrays of elementary cells, being in either an active or a passive state: at each time step the state of all the cells is updated depending on the state of the surrounding ones. Cellular automata then provide one with a test system the evolution of which can be studied by invoking a minimal set of rules, thus considerably reducing technical requirements on their simulation. Despite their simplicity, complex unpredictable behaviors can be observed, as in the celebrated example of Conway's game of life [10], or as illustrated in the extensive work of Wolfram (see [11,12]). Nowadays, cellular automata have found connections to random number generation [13], cryptography [14], and universal computing [15], and there even exist proposals for a unifying string theory based on this model [16].

Quantum features can be introduced as a way to gain insight on a larger class of fundamental effects. Indeed, the problem of reversibility is of striking importance in the foundations of the theory itself. While the evolution of a closed quantum system, expressed by a unitary transformation, is deterministic and invertible, the coupling with an unknown environment injects an uncontrolled element into the dynamics. The interaction with a thermal bath, in fact, establishes correlations with a system the state of which is unknown, which inevitably leads to the leak of information about the system under control, the purity of which typically decreases as it is driven to a mixed state. Quantum cellular automata (QCA) can then be defined by allowing for superposition states of their elementary cells [17-19]. Like their classical counterparts, QCA have been studied for their implications for quantum computing [20-24], but they have also found application to fundamental studies on quantum fields [25-28], to the simulation of quantum gases and 
quantum walks [29], and to the analysis of biological processes such as photosynthesis and eye vision [30].

Reversible cellular automata form a natural model of reversible computing. Also, quantum cellular automata require reversibility in order to simulate properly the Schrödinger equation, or any model in general that requires the conservation of physical quantities. In the literature there has been a considerable effort in proofs and simulations that account for reversibility in both CA and QCA. So far, however, tests on QCA coupled with a noisy environment are still missing. This is actually the most realistic and interesting case since any actual physical process will be affected by noise in some form, compromising its unitary evolution and hence its reversibility. For practical purposes, it is thus necessary to comprehend and control the role of quantum noise on the reversibility of the system.

Quantum walks [31-38] are closely related to QCA [29,39], since both architectures can explore the propagation of excitations and quantum information through networks; in this respect, it should be possible to have a unifying picture of classical and quantum evolutions by introducing tuneable noise. This has been accomplished in Ref. [40], where a suitable generalization of unitaries to generic maps is illustrated. It has been shown that excitation transfer in the QCA array can be continuously driven from the quantum to the classical stochastic regime. The presence of quantum coherence, in general, ensures a higher transfer efficiency in shorter times, sometimes with the assistance of noise [40]. Related studies have also examined quantum information processing via noisy QCA $[41,42]$. It should be noted that nonunitary quantum automata had already been introduced in Ref. [43].

These studies are part of an effort towards understanding how quantum properties may affect and possibly enhance transport in networks, with possible implications for biophysics [44]. On the other hand, such an interest in quantum coherence, compounded with the general framework of resource theories proper to quantum information science, has led to the introduction of suitable quantifiers for quantum coherence as a genuine resource [45-49].

In this paper, we illustrate the transition from reversible to irreversible dynamics, and from classical to quantum dynamics in the evolution of a linear-array QCA. In particular, we adopt the measures of quantum coherence introduced in Ref. [45] to introduce characteristic times for the loss of coherence, and find suitable quantifiers for a similar treatment of reversibility. These times are connected to the parameters describing the noise present in the evolution, as well as to the size of the automaton. Our studies found that in the noiseless case coherence oscillates in time with a spectrum of frequencies which depends on the size. In the presence of relevant classes of noise, namely, dephasing and amplitude damping, we witness a buildup of coherence at early times, followed by an exponential decay for long times. Remarkably, the decay of coherence is slower for increasing size. Furthermore, we comment on the effects of excitation localization in the reversibility that become manifest only in the presence of noise.

The paper is organized as follows. In Sec. II we introduce the model of noisy QCA investigated. In Sec. III we consider how the coherence evolves in time by tuning the dissipative effects. In Sec. IV we introduce a notion of reversibility for the noisy QCA and we see how noise affects the probability of restoring the initial state. Finally, in Sec. V, we state final considerations and draw conclusions.

\section{THE MODEL FOR NOISY QCA}

Despite the multitude of definitions used in literature to implement QCA, the model has four main features dating back to the early proposal of von Neumann [9].

(1) Discreteness. The automaton consists in a quantum lattice, in which every site is an independent physical system with a finite set of states of a Hilbert space. The state of each site evolves in discrete time steps through the application of a specific rule, which is usually taken to be unitary, but can be generalized to accommodate the presence of noise.

(2) Homogeneity. The rule is applied to every site at each time step and must be independent of lattice and time translations.

(3) Locality. Information must travel with finite speed through the lattice, hence the rule is applied only to a wellspecified neighborhood of each cell and its evolution at each step is independent from the rest of the lattice.

(4) Causality. The evolution is deterministic, in the sense that by knowing the configuration of the lattice at some time step it is possible to predict completely the configuration in the next step. In order for the update rule to be local and causal, it is essential to provide a convenient partitioning scheme, in which the lattice is divided in partitions of noninteracting neighboring blocks and the rule is applied to every block of a partition before considering the subsequent partition [50].

Our model of QCA is a one-dimensional array of $N$ twolevel nodes, being in either an excited or a ground state; these represent the elementary cells of the automaton. We consider here the case when a single excitation is present at the initial time $t=0$. This restricts our attention to the single-excitation sector: the possible classical states of the network, the ones in which the excitation is present at the $n$th site, are labeled as $|n\rangle$. For the dynamics to capture the essential features of the automata, it needs to satisfy the above-mentioned requirements of discreteness in time and space, homogeneity, locality, and causality: these are met if we partition the system in pairs of neighboring nodes, 1 and 2, 3 and 4, and so on (Fig. 1), and update the state of the QCA via unitary operations in the form

$$
U=\left(\begin{array}{cc}
\cos \theta & \sin \theta e^{i \phi_{2}} \\
-\sin \theta e^{i \phi_{1}} & \cos \theta e^{i\left(\phi_{1}+\phi_{2}\right)}
\end{array}\right)
$$

acting on the two-dimensional (qubit) subspace spanned by $|n\rangle$ and $|n+1\rangle$ for all the pairings above, which amounts to transferring the excitation with probability $\sin ^{2} \theta$ to the adjacent node, and leaving on the original node with probability $\cos ^{2} \theta$. This action is different from a simple stochastic transfer since phases are established between these two possibilities. An alternative parametrization can be obtained as

$$
U=\frac{1}{\sqrt{1-p+q}}\left(\begin{array}{cc}
\sqrt{1-p} & \sqrt{q} e^{i \phi_{2}} \\
\sqrt{q} e^{i \phi_{1}} & -\sqrt{1-p} e^{i\left(\phi_{1}+\phi_{2}\right)}
\end{array}\right)
$$




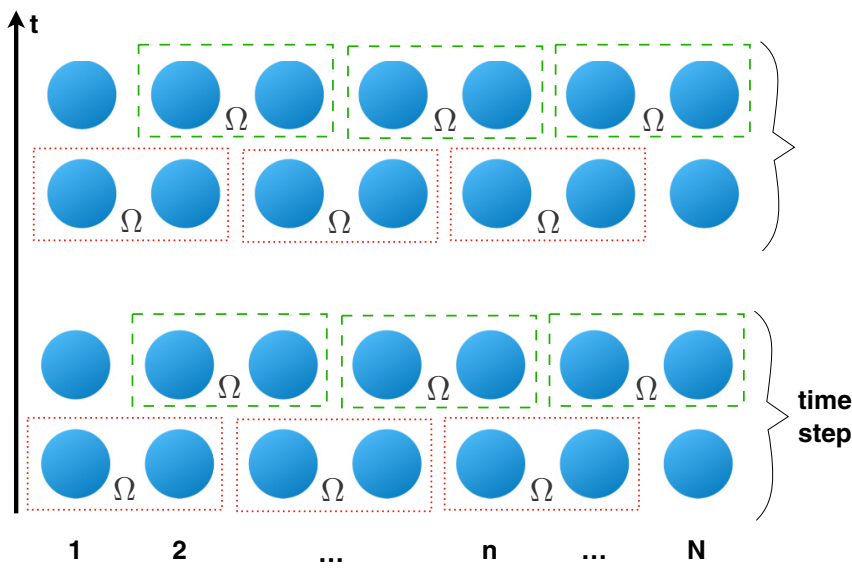

FIG. 1. One-dimensional quantum cellular automata. Each node is a two-level system, and excitations can be transferred between adjacent nodes by the action of the quantum map $\Omega$, that can be tuned from unitary to classical stochastic by the amount of noise present. A single time step of the full QCA evolution consists in replicating the action of $\Omega$ on the red pairings first (dotted boxed), and then on the green pairings (dashed boxes).

where $0 \leqslant p, q \leqslant 1$ can be interpreted as the probabilities of moving the excitation to the site to the left or to the right, respectively. The time step is completed by shifting the partitioning by one cell to the right (Fig. 1) and applying $U$ on the pairs of nodes 2 and 3, 4 and 5, etc., in such a way that the whole network is involved. Since excitations are neither created nor destroyed at any time, the state of the QCA always belongs to the single-excitation sector.

This purely unitary dynamics can be generalized to a dissipative one by introducing dephasing and amplitude damping to the qubit evolution; these have been shown to be sufficient to reproduce stochastic excitation transfer in the chain [40]. Dephasing is described by the map $\Phi_{\xi}$, characterized by a parameter $\xi$ describing the strength of the dephasing; the associated Kraus operators are

$$
D_{0}=\sqrt{1-\xi} \mathbb{1}, \quad D_{1}=\sqrt{\xi}\left(\mathbb{1}+\sigma_{z}\right) / 2, \quad D_{2}=\sqrt{\xi}\left(\sigma_{z}-\mathbb{1}\right),
$$

where $\sigma_{j}$ for $j=x, y, z$ stand for the Pauli matrices. Along with dephasing, we will also consider amplitude damping $\Xi_{\eta}$, with strength $\eta$, and associated operators

$$
L_{0, \eta}=\frac{\mathbb{1}+\sigma_{z}}{2}+\sqrt{1-\eta} \frac{\mathbb{1}-\sigma_{z}}{2}, \quad L_{1, \eta}=\sqrt{\eta} \frac{\sigma_{x}+i \sigma_{y}}{2} .
$$

While normally $0 \leqslant \eta \leqslant 1$, we can extend this to negative values $(-1 \leqslant \eta<0)$ as a shorthand notation for the inverted channel, i.e., the one with elements $\sigma_{x} L_{0,|\eta|} \sigma_{x}$, and $\sigma_{x} L_{1,|\eta|} \sigma_{x}$. Overall, the action of the complete map on a generic qubit state $\rho$ is written as

$$
\omega_{\xi, \eta}(\rho)=\Xi_{\eta}\left(\Phi_{\eta}\left(U \rho U^{\dagger}\right)\right)
$$

so that the evolution of the full network is the composition $\Omega_{\xi, \eta}$ of these maps applied to node pairs, as described above (Fig. 1):

$$
\rho(t+1)=\Omega_{\xi, \eta}(\rho(t))=\Omega_{\xi, \eta}^{t+1}(\rho(0)),
$$

where $\rho(t)$ is the state of the full network at time $t$. The map $\omega_{\xi, \eta}$ can also be expressed in terms of the probabilities $p$ and $q$, by observing that $\eta=p-q$, and $\cos (2 \theta)=(1-p-q) /$ $(1-|\eta|)$.

\section{COHERENCE}

The quantum coherence of the state $\rho$ in a certain basis can be quantified by means of the relative entropy with respect to the state $\rho^{D}$, which has the same populations as $\rho$ (i.e., the same diagonal entries in the selected basis), but all the off-diagonal terms set to zero [45]:

$$
C_{S}(\rho)=S\left(\rho \| \rho^{D}\right)=\operatorname{Tr}(\rho \ln \rho)-\operatorname{Tr}\left(\rho^{D} \ln \rho^{D}\right) .
$$

This implies that we have determined a privileged basis for decomposing the matrix $\rho$ on physical motivations; in our system this is naturally given by the $|n\rangle$ vectors. The measure $C_{S}(\rho)$ is then interpreted as the amount of information that is needed to learn $\rho$ if one has knowledge of its diagonal terms. Alternatively, one can build a measure based on the $l_{1}$ distance between $\rho$ and $\rho^{D}[45]$ :

$$
C_{1}(\rho)=\left\|\rho-\rho^{D}\right\|_{1}=\sum_{i \neq j}\left|\rho_{i, j}\right|,
$$

which is simply the magnitude of the off-diagonal terms. The notion of coherence can then be extended to quantum operations, by defining as incoherent those maps that cannot generate coherent states from incoherent states [51-54]. Both measures satisfy the requirements of vanishing for incoherent states, not increasing under incoherent operations, and representing a proper distance between $\rho$ and the closest incoherent state [45].

We start our analysis with the evolution in the absence of any decoherence effect, thus setting $\eta=0$, and $\xi=0$. The array is initialized in the state $|1\rangle$, i.e., the excitation is present only on the first node; at each time step, the full chain is therefore in a pure state $|\psi\rangle=\sum_{n} c_{n}|n\rangle$. The evolution of the coherence as a function of the time steps is illustrated in Fig. 2: the coherence reaches a limit value, in the presence of fast oscillations. As one might expect, the two measures (7) and (8) display similar behaviors, both of them increasing with the number of nodes $N$, the former with $\ln N$ and the latter with $N$. This is due to the fact that the dimension of the single-excitation subspace to which the evolution is constrained is $N$, while the maxima taken by the two measures over such a space are, respectively, $\ln (N)$ and $N-1$. Notice that this applies to the case $\theta=$ $\pi / 4$ which, corresponding to a balanced superposition at the single-qubit level, spreads out the coherence maximally over the chain and thus attains maximum coherence on the shortest time scale. The oscillations in the coherence are connected to the average position $\langle x\rangle=\sum_{n}\left|c_{n}\right|^{2} n$ of the excitation in the chain, with the minima of $C_{S}(|\psi\rangle\langle\psi|)$ and $C_{1}(|\psi\rangle\langle\psi|)$ occurring in correspondence of the extrema of $\langle x\rangle$. This is due to the fact that, when most of the population nears the chain's boundaries, the available direct-sum Hilbert space in the first excitation sector shrinks (simply because it does not extend beyond the end of the chain). Furthermore, we may observe 

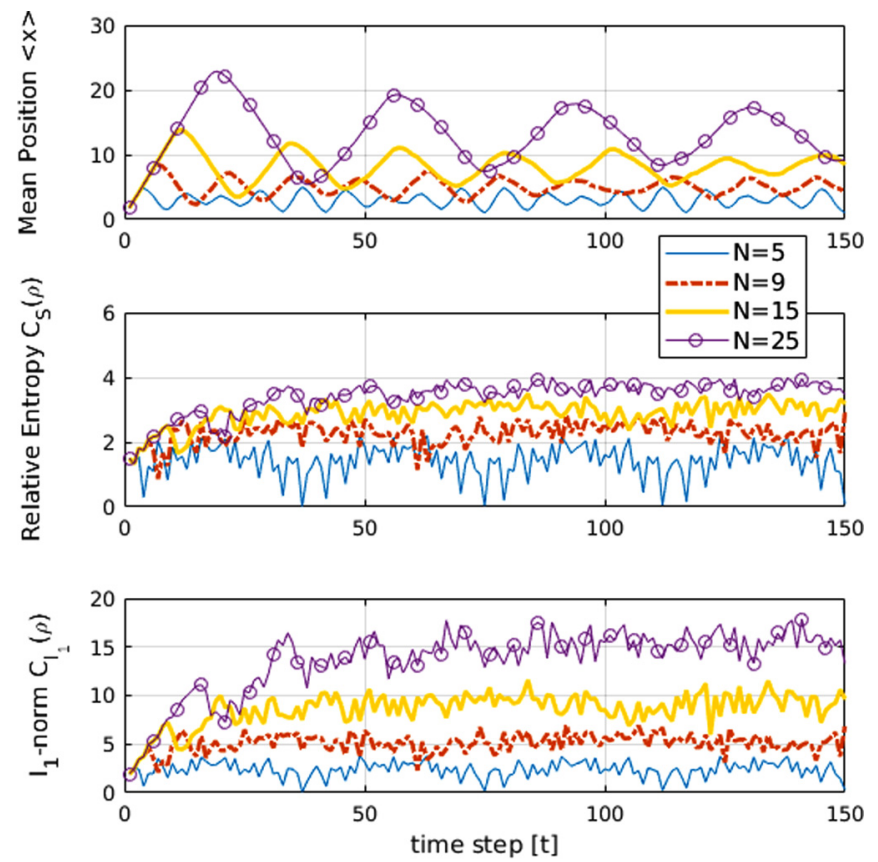

FIG. 2. Evolution of the quantum coherence of the noiseless QCA as a function of the time step for different lengths of the array. The unitary evolution considered corresponds to $\theta=\pi / 4$ in Eq. (2), the one giving the highest value. Upper panel: Average position of the excitation $\langle x\rangle$. Middle panel: Evolution of the entropy-based measure of coherence $C_{S}(|\psi\rangle\langle\psi|)$. Lower panel: Evolution of the $\mathrm{1}_{1}$-norm measure $C_{1}(|\psi\rangle\langle\psi|)$.

that $\langle x\rangle$ increases linearly until the excitation approaches the boundary for the first time; afterwards, one has a different dynamics where the mean position adopts a quasiperiodic behavior (a characteristic of finite quantum systems evolving under discrete time).

Since the two coherence measures give similar qualitative information, in the following we can focus on $C_{S}(\rho)$. It has been observed that for given coupling strengths in the unitary $U$ in Eq. (2), i.e., for a given $\theta$, the QCA present different behaviors depending on the sum of the phases $\phi_{1}+\phi_{2}$ : if the latter is zero, localization of the excitation might occur due to interference effects [40]. This is reflected in the coherence, as shown in Fig. 3: localization restricts the evolution of the system to a subset of its accessible states, thus limiting the maximal value of $C_{S}(|\psi\rangle\langle\psi|)$, and $C_{1}(|\psi\rangle\langle\psi|)$. In both cases, similar short-time oscillations occur.

We now turn our attention to how noise affects these behaviors; we will start by reporting the effect of the dephasing strength $\xi$, with no amplitude damping $\eta=0$. The QCA is initialized in the same pure state $|1\rangle$ as before, but now dephasing occurs during its evolution. At any time, the system will be in a mixture $\rho(t)$. When inspecting the coherence $C_{S}(\rho(t))$, a competition between two effects is observed, as shown in Fig. 4: while at short times coherence is built by the action of the unitary $U$, for longer times this starts being reduced by the presence of the noise. The average position of the excitation reflects this interplay: its oscillations are damped as coherence vanishes. The QCA are then driven to a long-term completely incoherent state, representing the thermal death of
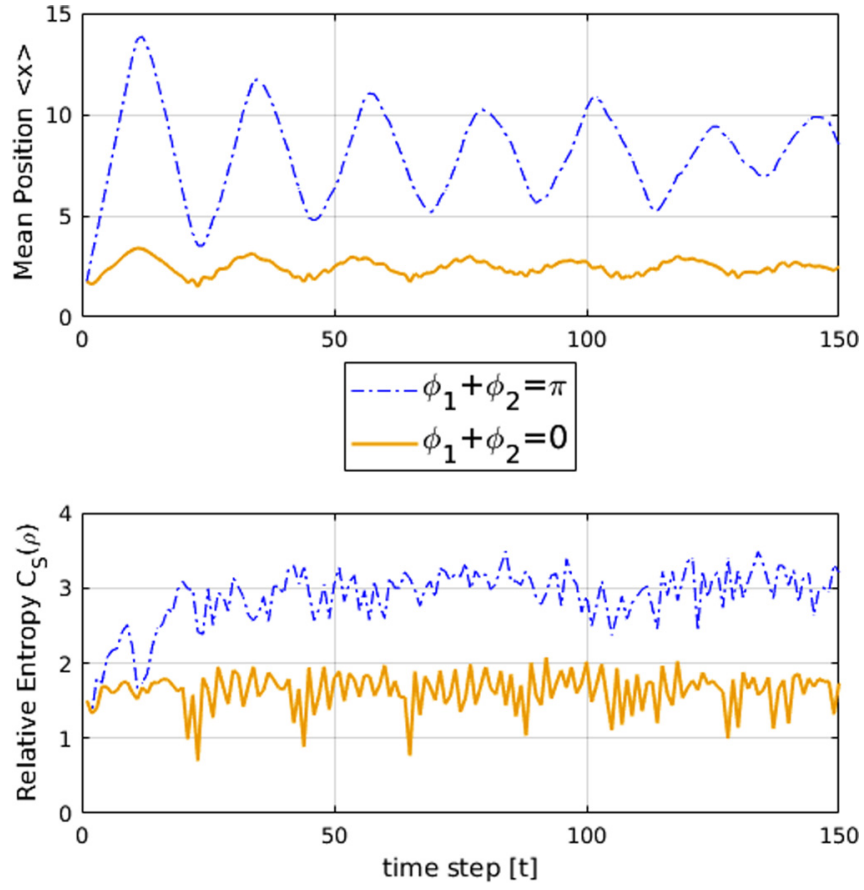

FIG. 3. The role of the phase $\phi_{1}+\phi_{2}$ in the production of quantum coherence, without noise and for $\theta=\pi / 4$. The length of the array is $N=15$. The occurrence of localized states in the array for $\phi_{1}+\phi_{2}=\pi$ prevents achieving maximal coherence, while for $\phi_{1}+\phi_{2}=0$ the localization mechanism is suppressed, and the QCA can explore its full space.

the system, as discussed in the next section. A characteristic time $T_{\text {dec }}$ for the decoherence can be estimated by fitting $C_{S}(\rho)$ with an exponential curve $\sim e^{-t / T_{\mathrm{dec}}}$. This is reported as a function of $\xi$ for different chain lengths $N$ (Fig. 5): the same
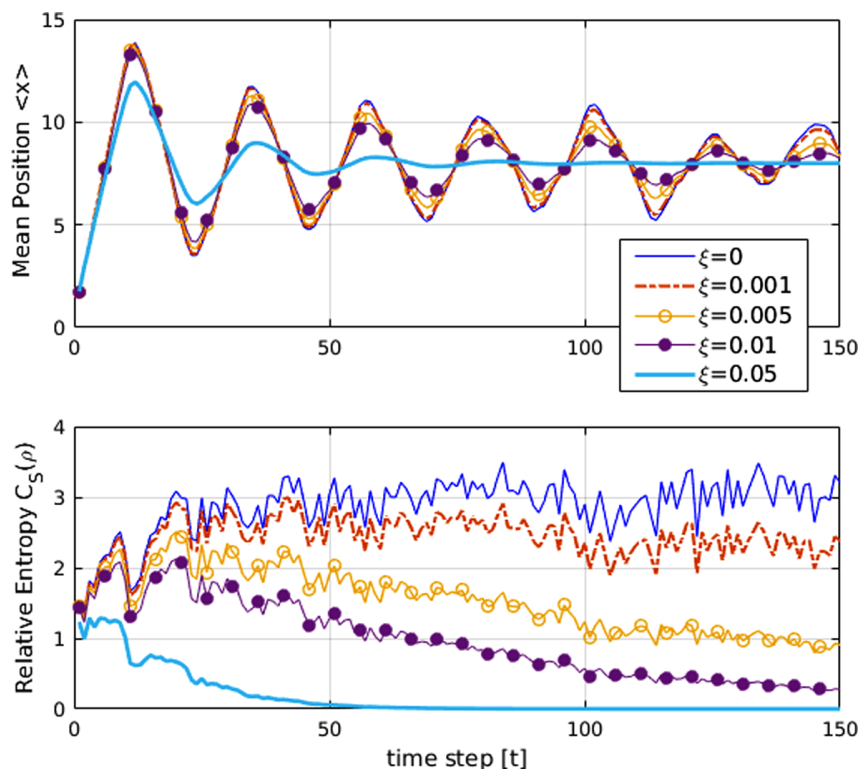

FIG. 4. Comparison of mean position of the excitation and level of coherence in the presence of pure dephasing. We have considered a chain of length $N=15$, with the phase in the unitary satisfying $\phi_{1}+\phi_{2}=\pi$ and $p=0.5$. 


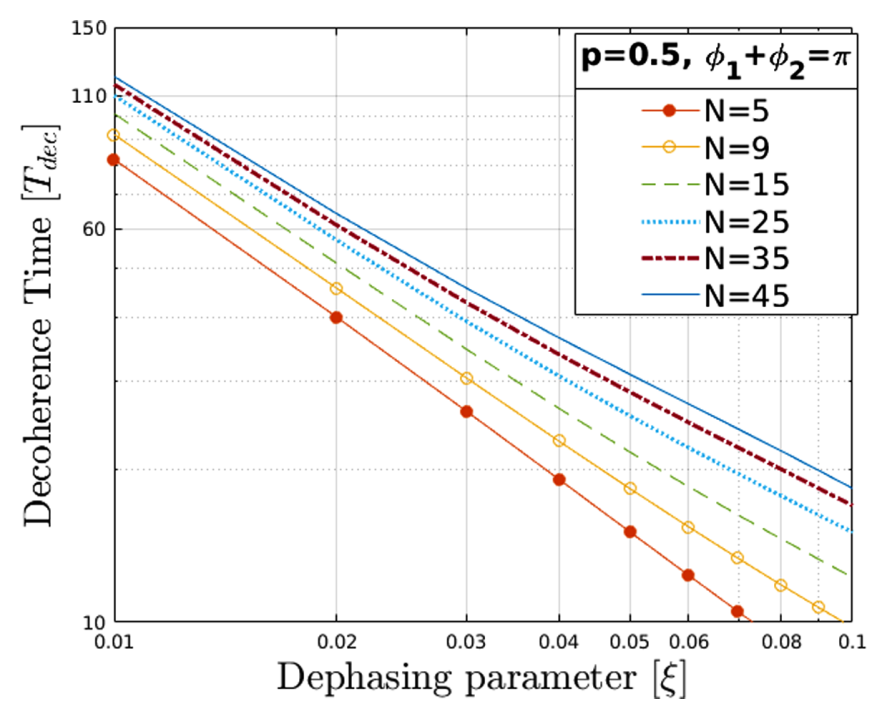

FIG. 5. Trend of decoherence times vs $\xi$ for various lengths of the chain, plotted in log-log scale.

level of noise results in a slower loss of coherence as the size of the QCA increases. This can be understood by considering the action of the dephasing Kraus operators on the direct sum Hilbert space that forms the single-excitation sector: on a single application of the map, only the off-diagonal elements pertaining to pairs of nearest-neighbor sites are damped, while the coherence between farther removed sites is not affected at all. Hence, coherence is damped only via the dephasing of the elements immediately above (and below) the main diagonal. Longer chains are therefore comparatively less affected by such a noise for the same strength. Notice that this still damps the overall coherence, since the off-diagonal elements farther away from the main diagonal must still build up from the damped ones through repeated applications of the unitary part of the local automaton.

Insight can be gained by inspecting the Fourier transform of $C_{S}(\rho(t))$, thus considering the pseudofrequency domain with respect to the evolution time (Fig. 6). In the noiseless case, the spectrum presents distinct peaks only for short array lengths. As the number of nodes increases, the spectrum starts presenting a large tail with rapid modulations. This reflects how correlations are established between nodes of any distance: for growing $N$ this results in multiple time scales being introduced in the problem. When adding the action of dephasing, the spectrum is smoothed, and low-frequency components are strongly suppressed.

Similar considerations can also be derived when inspecting the behavior under amplitude damping (we fix $\xi=0$ ): Fig. 7 illustrates that the automata present a very similar trend in their coherence as under dephasing, with the same peculiar distinction between constructive and destructive interference effects that lead, in the latter case, to localization, which is not disrupted by the presence of damping. We observe that the loss of coherence occurs rapidly even for modest levels of noise: in Figs. 4 and 7 it is evident how values of the noise parameters as low as 0.1 are sufficient to confine a coherent behavior to the first few tens of time steps. Therefore, as one should expect, coherent dynamics itself seems to be only relevant for "fast" processes.
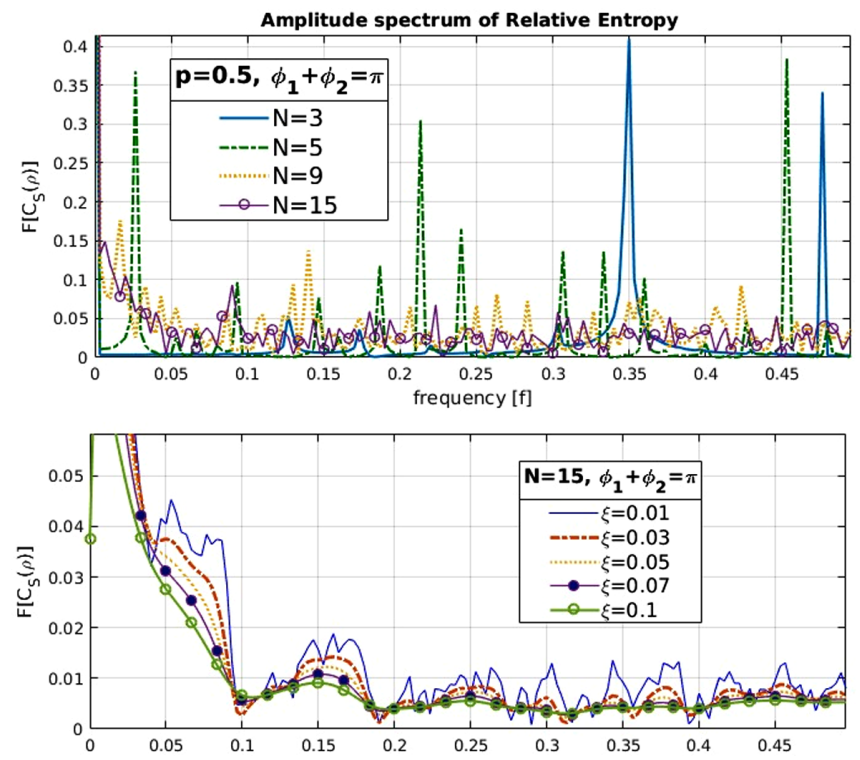

FIG. 6. Fast-Fourier transform of the coherence $C-S(\rho(t))$ in the noiseless (upper) and noisy (lower panel) case. Notice how increasing the size $N$ in the absence of noise leads to a complex structure of the spectrum, due to building of coherence terms at different time scales. In the presence of noise, long-term oscillations are inhibited by the presence of noise, and, in general, the shape of the spectrum is simplified.

\section{REVERSIBILITY}

Quantum mechanics simplifies the problem of reversibility in cellular automata. In the classical case the inverse of a cellular automaton is again a cellular automaton, provided that the rule is locally invertible, but it is a highly nontrivial matter to determine the neighborhood scheme of the inverse, which can be much larger than the neighborhood of the automaton itself. A general theorem on QCA, proved by Schumacher, ensures that any quantum cellular automaton is structurally reversible and the inverse of a nearest-neighbor quantum cellular automaton exists and is a nearest-neighbor quantum cellular automaton (see [17]). The theorem is based on the Margolus neighborhood scheme, which gives a privileged role to partitioned QCA [55]. In order to invert time in block partitioned QCA it is simply necessary to apply the inverse rule from the last block of the last partition to the first block of the first partition.

The evolution operator $\Omega_{\xi, \eta}$ respects the partitioning in noninteracting neighborhoods [40], and contains both unitary and dissipative contributions. The latter cannot be inverted; thus we will introduce, as the "Schumacher inverse," the operation $\tilde{\Omega}_{\xi, \eta}$, defined as the one with the unitary $\Omega_{0,0}$ inverted following Schumacher's construction, followed by phase damping and amplitude damping with the same strengths $\xi$ and $\eta$ as in the original automaton. In order to quantify the degree of reversibility, we let the system evolve for $T / 2$ time steps by $\Omega_{\xi, \eta}$, then invert the dynamics by applying $\tilde{\Omega}_{\xi, \eta}$ for the same number of steps; finally we consider the probability that the system ends in its initial state: $P_{1}(T)=$ $\operatorname{Tr}\left\{\rho_{0} \tilde{\Omega}_{\xi, \eta}^{T / 2}\left[\Omega_{\xi, \eta}^{T / 2}\left(\rho_{0}\right)\right]\right\}$. 

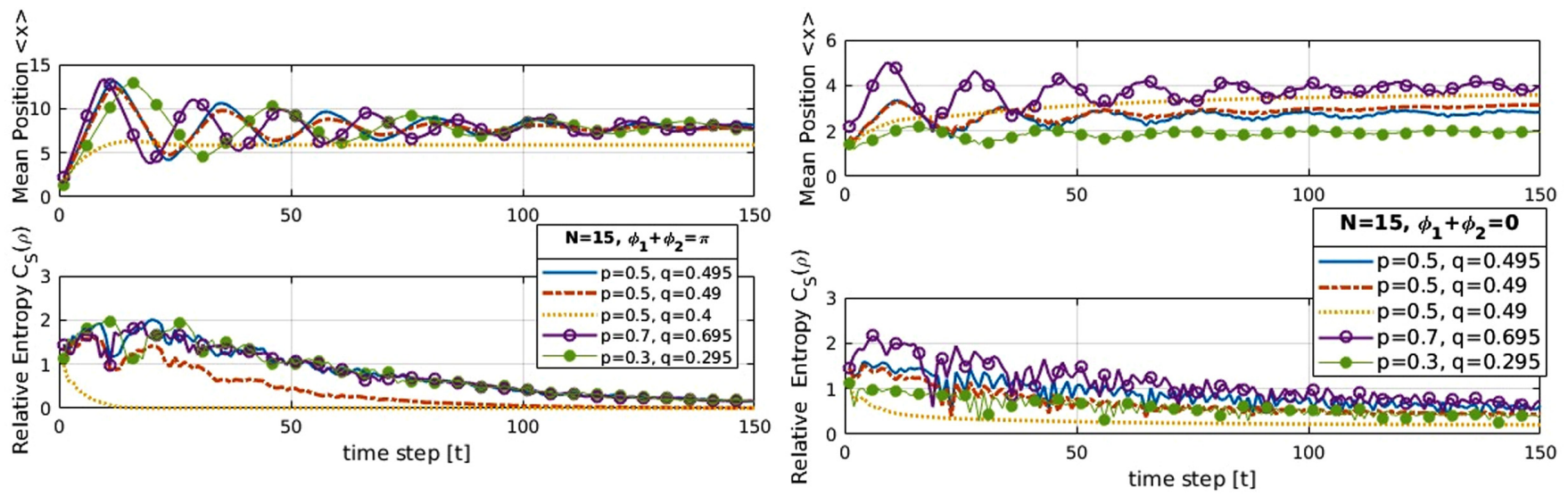

FIG. 7. Decoherence in the presence of amplitude damping. The plots report the mean position of the excitation, and the degree of coherence $C_{S}(\rho)$ without (left column) and with (right column) localization effects. The damping is expressed in terms of $\eta=p-q$.

Figure 8 summarizes the behavior of reversibility in the presence of dephasing, that rapidly drives the system towards irreversibility, leading it to the completely mixed state in the single-excitation sector. This can be easily verified by first noticing that the identity matrix $\mathbf{I} / N$ is a fixed point of the evolution since $\Omega_{\xi, 0}(\mathbf{I} / N)=\mathbf{I} / N$. Further, this is the only fixed point, as implied by Banach's fixed-point theorem: considering the trace distance $\mathcal{D}\left(\rho_{1}, \rho_{2}\right)$ between two arbitrary states in the single-excitation sector, it can be proved that the evolution map is contractive [56]:

$$
\mathcal{D}\left(\Omega_{\xi, 0}\left(\rho_{1}\right), \Omega_{\xi, 0}\left(\rho_{2}\right)\right) \leqslant \epsilon \mathcal{D}\left(\rho_{1}, \rho_{2}\right),
$$

where $0 \leqslant \epsilon<1$. Consequently, there exists a single fixed point, represented by the completely mixed state. Once the system reaches this maximum entropic state, we witness its thermal death, thus the quantum cellular automaton is not able to perform any computation nor to transmit information anymore: its arrow of time does not have a direction, since it is completely still. This condition is rapidly obtained, even in the presence of small levels of noise.

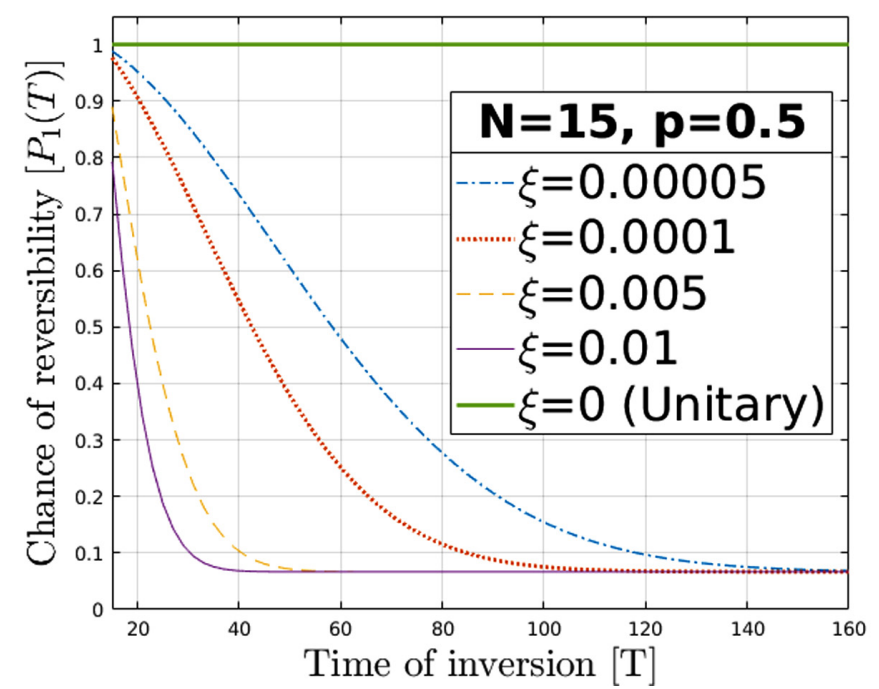

After a characteristic time, the probability of ending in the original state $\rho_{0}$ equals $1 / N$, i.e., the one obtained for a completely mixed state (Fig. 8). We can introduce an operative measure for an inversion time $T_{\text {irr }}$ as the one for which $P_{1}\left(T_{\text {irr }}\right) \simeq 1 / N$, to within a numerical threshold $\delta$ depending on the accuracy of the simulation; in our case, we choose $\delta=$ $10^{-4}$; the corresponding results are shown in Fig. 9. The time at which reversibility is completely lost depends loosely on the size of the chain: small-size effects ensure more robustness, even though for long chains the system behaves very similarly. Remarkably, the phases in the unitary $U$ in Eq. (2) set different limits to the reversibility, although in the pure limit $\xi=0$ perfect reversibility is clearly always achieved. Under the condition $\phi_{1}+\phi_{2}=\pi$, that leads to no localization of the excitation, a faster loss of reversibility is observed compared with the condition in the presence of localizations, which are, in fact, partly protected against the noise and retain the capability of walking back on their steps for longer times.

Decoherence under amplitude damping presents quantitative and qualitative difference with respect to the previous

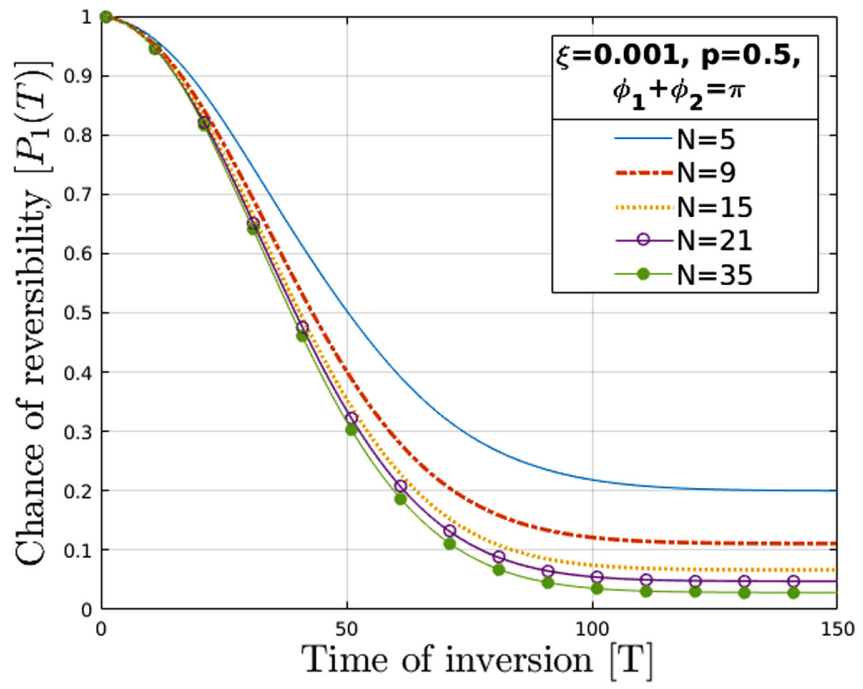

FIG. 8. Reversibility in the presence of pure dephasing. Left panel: The probability $P_{1}$ of inverting the dynamics and end in the initial state $\rho_{0}$, as a function of the total evolution time $T$, for different values of the dephasing parameter $\xi$. Right panel: Same as above, for different lengths $N$ of the chain. 

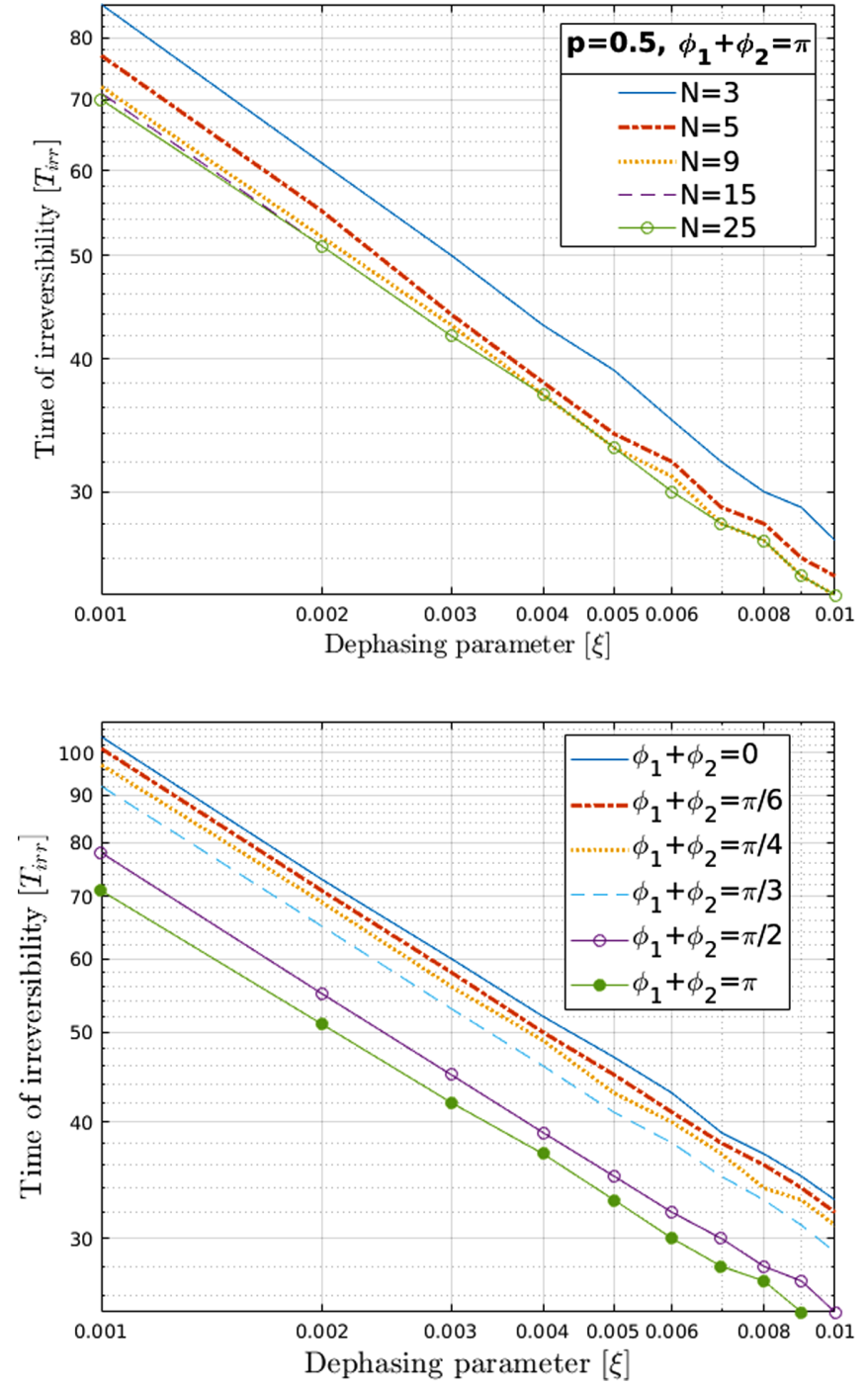

FIG. 9. Characteristic time for the occurrence of irreversibility of the system $T_{\text {irr }}$ in the presence of pure dephasing. Upper panel: $T_{\text {irr }}$ as a function of the dephasing parameter $\xi$ for different lengths $N$. Lower panel: Same as above, but for different phases $\phi_{1}+\phi_{2}$.

case of dephasing. However, as illustrated in Fig. 10, the presence of localization is always relevant to reversibility. In its absence, the loss reversibility occurs with a largely size-independent characteristic time. However, the final state of the evolution does not correspond to the completely mixed state, due to the population transfer operated by the noise: the probability $P_{1}(T)$ is always larger than the one for $\mathbf{I} / N$. For long times, there is an equilibrium between noisy and unitary transfer mechanisms. Small-size effects are manifested as small oscillations, which are rapidly damped if the size is increased. Localization changes this behavior: the probability $P_{1}(T)$ is now considerably above the mixed-state limit, even for long chains. This is due to the fact that it is easier for the noise to pump population into the first site starting from a localized state than it is from a delocalized situation. Qualitatively, the increase in the long-time value of $P_{1}(T)$ can be understood by noticing that amplitude damping in part freezes the evolution of the system by forcing it to remain in the initial state: the

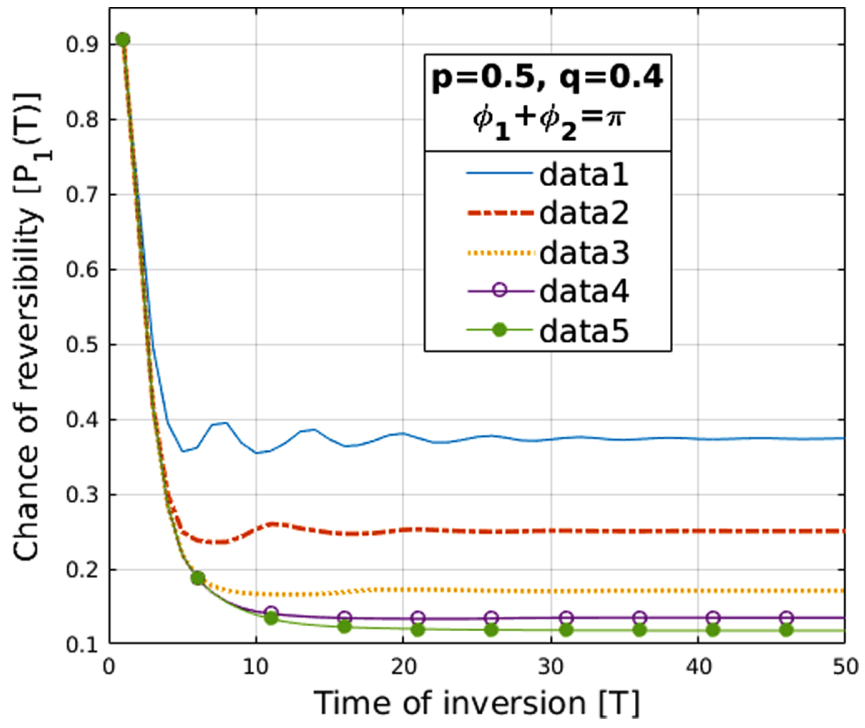

FIG. 10. Reversibility in the presence of amplitude damping. Left panel: The probability $P_{1}$ of inverting the dynamics and end in the initial state $\rho_{0}$ as function of the total evolution time $T$ for phases $\phi_{1}+\phi_{2}=\pi$. Right panel: Same as above, but with $\phi_{1}+\phi_{2}=0$.

system retains some of its reversibility since it has not actually undergone any evolution.

\section{CONCLUSIONS}

In conclusion, we have studied the evolution of coherence as well as introduced a notion of reversibility for noisy quantum cellular automata. We have used the latter to inspect how noisy processes affect the probability of restoring the initial state following inversion of the evolution. We have used it to define a characteristic time for the system to achieve a steady state, and we have illustrated how different classes of noise process introduce qualitative difference in the reversibility of such steady states. Furthermore, we considered the effects of excitation localizations on reversibility that become manifest only in the presence of noise.

We remark how these two aspects evolve differently under the same noise levels, with coherence being more resilient. This is due, in part, to the fact that our definition for reversibility demands a second evolution via the Schumacher inverse. Although coherence is built in the forward process, it is consumed when inverting, and is unable to drive the system back on its path. This is also reflected in the different behaviors of coherence and reversibility in terms of the chain length $N$ : smaller chains maintain coherence for less time than longer ones, but, also due to their small size, their evolution is easier to invert, as a comparison between Figs. 5 and 9 reveals.

\section{ACKNOWLEDGMENT}

We thank P. Mataloni, M. G. A. Paris, and M. Avalle for discussion and encouragement. 
[1] J. L. Lebowitz, Boltzmann's entropy and time's arrow, Phys. Today 46(9), 32 (1993).

[2] H. D. Zeh, The Physical Basis of the Direction of Time (Springer, New York, 2007).

[3] A. Eddington, The Nature of the Physical World (MacMillan, London, 1928).

[4] L. Boltzmann, Entgegnung auf die wärmetheoretischen Betrachtungen des Hrn. E. Zermelo, Ann. Phys. (Leipzig) 293, 773 (1896) [translated and reprinted in S. G. Brush, Kinetic Theory 2 (Pergamon, New York, 1966)].

[5] C. Gogolin and J. Eisert, Equilibration, thermalisation, and the emergence of statistical mechanics in closed quantum systems, Rep. Prog. Phys. 79, 056001 (2016).

[6] D. Andrieux, P. Gaspard, S. Ciliberto, N. Garnier, S. Joubaud, and A. Petrosyan, Entropy Production and Time Asymmetry in Nonequilibrium Fluctuations, Phys. Rev. Lett. 98, 150601 (2007).

[7] T. Batalhão, A. Souza, R. Sarthour, I. Oliveira, M. Paternostro, E. Lutz, and R. Serra, Irreversibility and the Arrow of Time in a Quenched Quantum System, Phys. Rev. Lett. 115, 190601 (2015).

[8] A. Hofmann, V. F. Maisi, J. Basset, C. Reichl, W. Wegscheider, T. Ihn, K. Ensslin, and C. Jarzynski, Heat dissipation and fluctuations in a driven quantum dot, Phys. Status Solidi B 254, 1600546 (2017).

[9] J. von Neumann and A. W. Burks, Theory of Self-Reproducing Automata (University of Illinois Press, Champaign, 1966). The original idea is due to von Neumann in the early 1940s, but the book was completed by Burks only in 1966.

[10] M. Gardner, Mathematical games: The fantastic combinations of John Conway's new solitaire game "life," Sci. Am. 223, 120 (1970).

[11] S. Wolfram, Statistical mechanics of cellular automata, Rev. Mod. Phys. 55, 601 (1983).

[12] S. Aaronson, A new kind of science, Quantum Inf. Comp. 2, 410 (2002).

[13] Wolfram Mathematica 11.3, Documentation on Random Number Generation (Wolfram Research Inc., 2017), see http:// support.wolfram.com/kb/472.

[14] S. Wolfram, Cryptography with cellular automata, in Proceedings of the Advances in Cryptology - CRYPTO 1985, edited by H. C. Williams, Lecture Notes in Computer Science, Vol. 218 (Springer, Berlin, Heidelberg, 1986), pp. 429-432.

[15] M. Cook, Universality in elementary cellular automata, Complex Syst. 15, 1 (2004).

[16] G. 't Hooft, Discreteness and determinism in superstrings, arXiv:1207.3612.

[17] B. Schumacher and R. F. Werner, Reversible quantum cellular automata, arXiv:quant-ph/0405174.

[18] J. Watrous, On one-dimensional quantum cellular automata, in Proceedings of IEEE 36th Annual Foundations of Computer Science (IEEE, New York, 1995), pp. 528-537.

[19] A. Kondacs and J. Watrous, On the power of quantum finite state automata, Proceedings of the 38th Annual Symposium on Foundations of Computer Science, 1997 (unpublished).

[20] R. Raussendorf, Quantum cellular automaton for universal quantum computation, Phys. Rev. A 72, 022301 (2005).
[21] K. G. H. Vollbrecht and J. I. Cirac, Reversible universal quantum computation within translation-invariant systems, Phys. Rev. A 73, 012324 (2006).

[22] D. Nagaj and P. Wocjan, Hamiltonian quantum cellular automata in one dimension, Phys. Rev. A 78, 032311 (2008).

[23] P. Arrighi and J. Grattage, Partitioned quantum cellular automata are intrinsically universal, Natural Computing 11, 13 (2012).

[24] G. M. D'Ariano, C. Macchiavello, and M. Rossi, Quantum cloning by cellular automata, Phys. Rev. A 87, 032337 (2013).

[25] S. Lloyd, A theory of quantum gravity based on quantum computation, arXiv:quant-ph/0501135.

[26] P. Arrighi, S. Facchini, and M. Forets, Discrete Lorentz covariance for quantum walks and quantum cellular automata, New J. Phys. 16, 093007 (2014).

[27] G. M. D'Ariano, N. Mosco, P. Perinotti, and A. Tosini, Pathintegral solution of the one-dimensional Dirac quantum cellular automaton, Phys. Lett. A 378, 3165 (2014).

[28] A. Bisio, G. M. D’Ariano, and A. Tosini, Quantum field as a quantum cellular automaton: The Dirac free evolution in one dimension, Ann. Phys. 354, 244 (2015).

[29] P. C. S. Costa, R. Portugal, and F. de Melo, Quantum walks via quantum cellular automata, arXiv:1803.02176.

[30] M. Mohseni, Y. Omar, G. S. Engel, and M. B. Plenio, Quantum Effects in Biology (Cambridge University, Cambridge, England, 2014).

[31] Y. Aharonov, L. Davidovich, and N. Zagury, Quantum random walks, Phys. Rev. A 48, 1687 (1993).

[32] H. B. Perets, Y. Lahini, F. Pozzi, M. Sorel, R. Morandotti, and Y. Silberberg, Realization of Quantum Walks with Negligible Decoherence in Waveguide Lattices, Phys. Rev. Lett. 100, 170506 (2008).

[33] A. Peruzzo et al., Quantum walks of correlated photons, Science 329, 1500 (2010).

[34] A. Schreiber et al., A 2D quantum walk simulation of twoparticle dynamics, Science 336, 55 (2012).

[35] T. Kitagawa et al., Observation of topologically protected bound states in photonic quantum walks, Nat. Comms 3, 882 (2012).

[36] A. Crespi et al., Anderson localization of entangled photons in an integrated quantum walk, Nat. Photonics 7, 322 (2013).

[37] F. Cardano et al., Quantum walks and wavepacket dynamics on a lattice with twisted photons, Sci. Adv. 1, e1500087 (2015).

[38] H. Defienne, M. Barbieri, I. A. Walmsley, B. J. Smith, and S. Gigan, Two-photon quantum walk in a multimode fiber, Sci. Adv. 2, 1501054 (2016).

[39] A. Bisio, G. M. D'Ariano, and P. Perinotti, Quantum walks, Weyl equation and the Lorentz group, Found. Phys. 47, 1065 (2017).

[40] M. Avalle and A. Serafini, Noisy Quantum Cellular Automata for Quantum Versus Classical Excitation Transfer, Phys. Rev. Lett. 112, 170403 (2014).

[41] M. Avalle, M. G. Genoni, and A. Serafini, Quantum state transfer through noisy quantum cellular automata, J. Phys. A 48, 195304 (2015).

[42] D. M. McNally and J. P. Clemens, Performance of 1D quantum cellular automata in the presence of error, AIP Advances 6, 095115 (2016).

[43] G. K. Brennen and J. E. Williams, Entanglement dynamics in one-dimensional quantum cellular automata, Phys. Rev. A 68, 042311 (2003). 
[44] F. Caruso, A. W. Chin, A. Datta, S. F. Huelga, and M. B. Plenio, Highly efficient energy excitation transfer in light-harvesting complexes: The fundamental role of noise-assisted transport, J. Chem. Phys. 131, 105106 (2009).

[45] T. Baumgratz, M. Cramer, and M. B. Plenio, Quantifying Coherence, Phys. Rev. Lett. 113, 140401 (2014).

[46] F. Levi and F. Mintert, A quantitative theory of coherent delocalization, New J. Phys. 16, 033007 (2014).

[47] A. Streltsov, U. Singh, H. S. Dhar, M. N. Bera, and G. Adesso, Measuring Quantum Coherence with Entanglement, Phys. Rev. Lett. 115, 020403 (2015).

[48] A. Winter and D. Yang, Operational Resource Theory of Coherence, Phys. Rev. Lett. 116, 120404 (2016).

[49] A. Streltsov, G. Adesso, and M. B. Plenio, Colloquium: Quantum coherence as a resource, Rev. Mod. Phys. 89, 041003 (2017).
[50] J. L. Schiff, Cellular Automata: A Discrete View of the World (Wiley, New York, 2008)

[51] J. Åberg, Quantifying superposition, arXiv:quant-ph/0612146.

[52] E. Chitambar and G. Gour, Comparison of incoherent operations and measures of coherence, Phys. Rev. A 94, 052336 (2016).

[53] E. Chitambar and G. Gour, Critical Examination of Incoherent Operations and a Physically Consistent Resource Theory of Quantum Coherence, Phys. Rev. Lett. 117, 030401 (2016).

[54] I. Marvian and R. W. Spekkens, How to quantify coherence: Distinguishing speakable and unspeakable notions, Phys. Rev. A 94, 052324 (2016).

[55] T. Toffoli and N. Margolus, Cellular Automata Machines: A New Environment for Modeling (MIT, Cambridge, MA, 1987).

[56] M. A. Nielsen and I. L. Chuang, Quantum Computation and Quantum Information, 2nd ed. (Cambridge University, Cambridge, England, 2010). 